\title{
O POTENCIAL EVOCADO COGNITIVO EM ADULTOS NORMAIS
}

\author{
Glaucio M. Franco'
}

\begin{abstract}
RESUMO - O objetivo deste estudo foi avaliar os resultados do potencial cognitivo auditivo em um grupo de adultos normais. Os testes foram realizados registrando-se a resposta evocada em $\mathrm{Cz}$ e Fz. Os dados obtidos foram analisados estatisticamente. São descritas neste trabalho as latências médias de N1, P2, P2AP, N2 e P3, a amplitude de P3, bem como o desvio padrão destas variáveis. Comentam-se algumas dificuldades encontradas na interpretação do teste destacando as limitações de seu uso clínico.
\end{abstract}

PALAVRAS-CHAVE: P300, potencial cognitivo, potencial endógeno.

The cognitive potential in normal adults

\begin{abstract}
The objective of this study was to evaluate the results of the auditory cognitive potential in a group of normal adults. The evoked response was obtained at $\mathrm{Cz}$ and $\mathrm{Fz}$. The data collected were statistically analysed. The mean latencies of N1, P2, P2AP, N2, P3 and amplitude of P3, as well the standard deviation of these variables were presented. We briefly comment on some pitfalls related to interpretation of the results as well as the limitations of the tecnique as a diagnostic tool.
\end{abstract}

KEY WORDS: P300, cognitive potentials, endogenous potentials.

Os potenciais evocados são obtidos através do registro e promediação das respostas aos estímulos sensoriais captados na superfície do crânio. Os potenciais evocados de longa latência, em especial os potenciais cognitivos (PC), são diretamente influenciados pela motivação do paciente, seu nível de atenção e experiência prévia. O PC é também denominado potencial endógeno ou potencial relacionado a eventos. Podemos obter PC com estímulos sensoriais de diversas naturezas; rotineiramente são utilizados os estímulos auditivos ${ }^{1,2}$. Os PC podem ser registrados quando da estimulação sensorial com dois diferentes estímulos da mesma modalidade, porém, com características físicas diferentes. Um dos estímulos é apresentado frequentemente, e o outro apenas ocasionalmente, em intervalos aleatórios (ODD BALL PARADYGM). O equipamento registra em separado a atividade cerebral evocada pelo estímulo frequente (estímulo ignorado) e a atividade evocada pelo estímulo raro ( objeto da atenção do examinando) ${ }^{1,2}$.

Este estudo analisa o P300 em um grupo de adultos normais.

\section{MÉTODO}

O grupo estudado era composto por 25 voluntários normais (9 homens e 15 mulheres) cujas idades variavam de 22 a 58 anos (média de idade 38 anos). Através de questionário objetivo e exame clínico foram descartados os voluntários acometidos por enfermidades sistêmicas. Foram também recusados aqueles com antecedentes de trauma crânio-encefálico e doença neurológica ou psiquiátrica. O uso ocasional de bebida alcoólica ou de medicação tranquilizante foi tolerado, desde que tivesse ocorrido mais de $48 \mathrm{~h}$ antes da realização do teste. Os exames foram realizados em sala silenciosa, em posição semi-sentada, com os olhos abertos, estando presentes apenas o examinando e o examinador. Foram utilizados fones com acolchoamento periauricular em ambas as orelhas. Os eletrodos ativos foram colocados em $\mathrm{Cz}$ e Fz e conectados ao "İNPUT 1" do amplificador. Os eletrodos de referência foram colocados no lado interno dos lóbulos da orelha (A1 e A2), interligados e conectados ao "INPUT" 2 do amplificador (Fig 1). O "terra" foi colocado em Fpz .

Como estímulos auditivos foram utilizados tons de 1 $\mathrm{KHz}$ para o estímulo frequente e de $2 \mathrm{KHz}$ para o estímulo raro. Foram feitos $20 \%$ de estímulos raros, randomicamente mesclados aos estímulos frequentes. O programa utilizado no presente trabalho não analisava o estímulo

Estudo realizado no Serviço de Neurofisiologia Clinica da Santa Casa de Misericórdia de Juiz de Fora: ${ }^{1}$ Professor Adjunto de NeurologiaRegente de Disciplina da Universidade Federal de Juiz de Fora (UFJF)

Recebido 21 Setembro 2000, recebido na forma final 12 Dezembro 2000. Aceito 14 Dezembro 2000.

Dr. Glauco Mendes Franco - Rua Oscar Vidal 245/1502 - 36016-290 Juiz de Fora MG - Brasil. E-mail: glauciof@artnet.com.br 


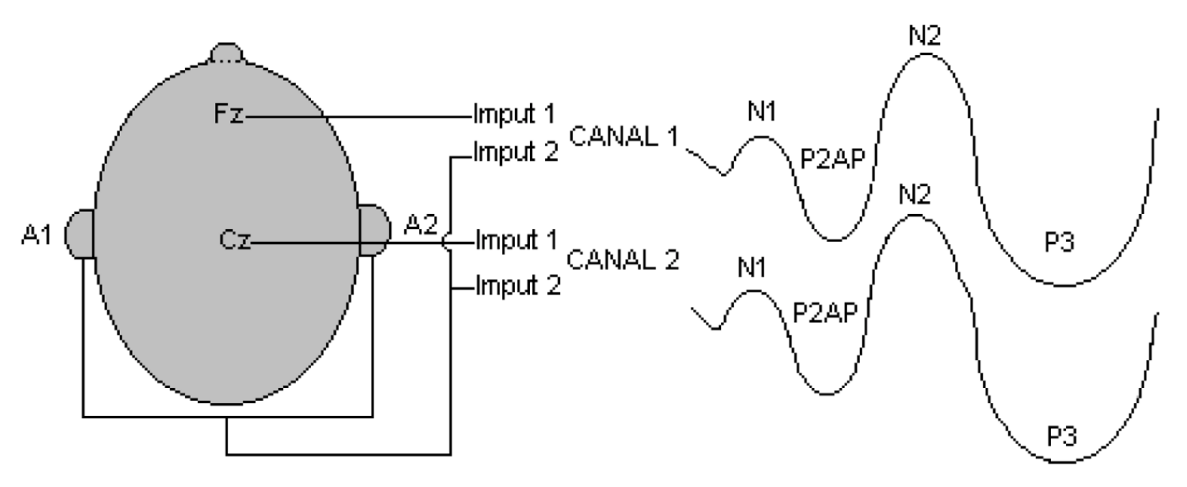

A posição dos eletrodos segue o modelo do sistema 10-20. Os principais componentes do PC são visualizados em canais separados para Cz e Fz.

Fig 1. Desenho esquemático: posição dos eletrodos e caracteristícas do traçado normal.

raro quando, na série, aleatória ele era o primeiro a ocorrer. $\mathrm{Na}$ eventualidade do estímulo raro ocorrer de modo sucessivo, apenas o primeiro estímulo da série era promediado. Solicitou-se ao examinando que identificasse o estímulo raro contando mentalmente o número de sua ocorrência. Os estímulos frequentes deviam ser ignorados. Terminada a série, o número de estímulos raros devia ser informado ao examinador. Com este procedimento pode-se avaliar a efetiva participação do examinando. Todos os testes foram realizados com duas passagens sucessivas que permitissem boa definição e comprovassem boa replicação. A identificação do complexo N2-P3 simultaneamente em Cz e Fz, foi considerada condição obrigatória para a aceitação dos resultados. Os gráficos obtidos foram registrados em disquete e papel termo-sensível.

Quando a morfologia do complexo N2-P3 apresentava-se com modulações que dificultavam a correta identificação das latências, optamos por adotar na análise do PC a técnica descrita por Chiappa para determinar o ponto de medida da latência em situações semelhantes e que ocorrem com o P100 do potencial evocado visual-padrão reverso ${ }^{3}$. A utilização desta técnica no PC é citada por Oken ${ }^{1}$.

O método consiste em traçar linhas tangentes aos segmentos descendente e ascendente do componente em estudo. Os prolongamentos destas linhas se cruzam em um ponto que serve de referência a uma perpendicular ao eixo do tempo. A latência corresponde ao ponto em que a linha perpendicular cruza o eixo da variável tempo (Fig 2).

\section{RESULTADOS}

Foram estudadas as latências referentes ao estímulo frequente e ao estímulo raro. De cada variável obtivemos inicialmente a média aritmética das duas passagens, o que constituiu o valor obtido daquela variável para cada voluntário. Os valores assim obtidos foram utilizados para a obtenção da média ge-

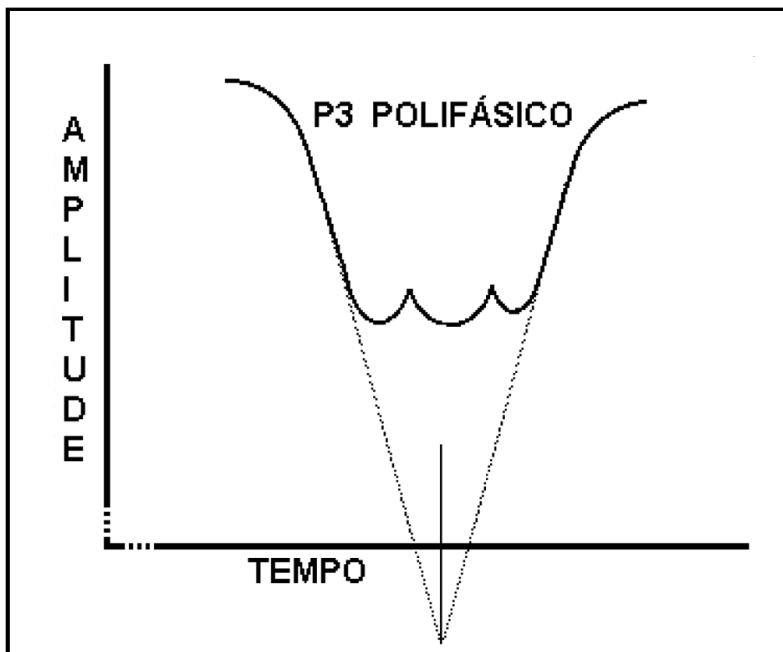

As linhas pontilhadas são tangentes aos segmentos ascendente e descendente de P3. A linha vertical partindo do ponto de encontro das tangentes determina, na linha horizontal (Tempo), o ponto onde se mede a latência.

Fig 2. Método de determinação da latência de P3.

ral da amostra e a determinação dos desvios padrão (Tabela).

Destacamos que os nossos resultados, coincidentes com os dados da literatura, demonstram uma grande variação nas latências do complexo N2-P3, em $\mathrm{Cz}$ e Fz, como evidenciado pelos altos valores do desvio padrão. A amplitude de P3, medida em relação a N2, revelou também grande variabilidade sobretudo em Fz. Registramos em Cz a amplitude média de 16,56 microvolts (desvio padrão de 5.92) e em Fz a amplitude média de 14,80 microvolts (desvio padrão de 8,16$)$. 
Tabela. Variáveis de tempo do potencial auditivo P300 em grupo de 25 adultos normais.

\begin{tabular}{lcccc}
\hline & \multicolumn{2}{c}{ Eletrodo $\mathrm{Fz}$} & \multicolumn{2}{c}{ Eletrodo Cz } \\
\hline Onda & $\mathrm{M}$ & $\mathrm{DP}$ & $\mathrm{M}$ & $\mathrm{DP}$ \\
$\mathrm{N} 1 \mathrm{~F}$ & 89.68 & 12.40 & 90.32 & 8.10 \\
P2 & 185.84 & 22.79 & 180.40 & 18.23 \\
N1R & 92.16 & 13.60 & 91.36 & 8.14 \\
P2AP & 166.68 & 11.70 & 163.04 & 12.37 \\
N2 & 218.44 & 22.32 & 274.72 & 7.71 \\
P3 & 321.24 & 33.59 & 315.08 & 25.50 \\
\hline
\end{tabular}

M, Média do grupo de voluntários para cada variável; DP, Desvio padrão; $\mathrm{N} 1 \mathrm{~F}$, Onda N1, estímulo frequente; $\mathrm{P} 2$, Onda P2, estímulo frequente; N1R, Onda N1, estímulo raro; P2AP, Onda P2 aparente, estímulo raro; N2, Onda $\mathrm{N} 2$, estímulo raro; $\mathrm{P} 3$, Onda P3, estímulo raro.

\section{DISCUSSÃO}

As enfermidades que acometem o encéfalo, com frequência produzem distúrbios das funções cognitivas. Os métodos neurofisiológicos não estudam alterações estruturais, registram os processos neurofisiológicos subjacentes, no tempo em que estão acontecendo. Embora sujeitos a variações e muitas vezes de difícil interpretação, estes potenciais têm a indiscutível qualidade de fornecer informações sobre o tecido nervoso vivo ${ }^{4}$. Os potenciais evocados relacionados a eventos possibilitam uma avaliação neurofisiológica da função cognitiva ${ }^{5,6}$. Sua descrição por Sutton (1965) ${ }^{7}$, trouxe grandes esperanças de que sua utilização clínica se tornasse método confiável para a avaliação de distúrbios comportamentais. Através de técnica de fácil execução é possível identificar disfunções na realização de tarefas mentais simples. $O$ fato de poder ser influenciado por disfunções encefálicas mas também paralela e simultaneamente, por situações fisiológicas ou pela atitude psicológica do examinando, tornou entretanto problemática a utilização do PC na prática clínica ${ }^{1,2}$.

Sua principal indicação está relacionada à avaliação das fases iniciais das demências e sua diferenciação das depressões ${ }^{8,9}$. Os PC podem apresentar variação de 15 a $20 \mathrm{~ms}$ na resposta $\mathrm{P} 3$ quando são realizadas, no mesmo paciente, exames com intervalos relativamente curtos. Por outro lado, têm sido relatados como normais valores entre 250 até 600 $\mathrm{ms}^{1}$. Por vezes o complexo N2-P3 não é identificado, mesmo na ausência de qualquer patologia 5 . A progressão da idade geralmente determina tendência ao aumento da latência de 1 a 1.5 ms por ano e a diminuição da amplitude ${ }^{10}$. Alguns autores relataram que, depois dos 45 anos, a progressão da latência se acentua, mas isto não foi confirmado ${ }^{11}$.

Ao utilizar os resultados do estudo de grupos de voluntários normais como padrão comparativo visando detectar anormalidades, devemos ter em mente os dados relatados acima, além de algumas outras considerações básicas.

Como nosso objetivo é comparar um grupo de indivíduos normais com um grupo homogêneo de pacientes o estudo comparativo poderá contribuir para identificar possíveis alterações de variáveis que se relacionam com a enfermidade em estudo. O método presta-se portanto, ao uso como ferramenta de pesquisa na comparação de dois grupos de constituição semelhante mas que tenham uma característica distintiva que se deseja testar estatisticamente $^{4}$. Os valores descritos anteriormente são válidos para o grupo estudado e não devem ser tomados genericamente como valores de normalidade para o método.

$\mathrm{Na}$ eventualidade de comparar os resultados de um grupo de pessoas normais com os resultados de um único paciente, a fidelidade do método é pequena e qualquer alteração deve ser interpretada com grande cuidado 4 . O valor do potencial cognitivo encontrado em um indivíduo isolado não tem valor diagnóstico ${ }^{4}$. Quando eventualmente utilizado, este método deve ser entendido como exame meramente auxiliar, sempre subordinado às características clínicas do caso e às observações obtidas em exames neuropsicológicos apurados.

\section{REFERÊNCIAS}

1. Oken BS. Endogenous event-related potentials. In Chiappa KH (ed). Evoked potentials in clinical medicine. Philadelphia: Lippincott-Raven, 1997:529-564.

2. Mauguiere F. Evoked Potentials . In Osselton JW (ed). Clinical neurophysiology. Oxford: ButterworthHeineman, 1995:325-532.

3. Chiappa KH. Pattern-shift visual evoked potentials: methodology. In Chiappa K H (ed) evoked potentials in clinical medicine. Philadelphia: Lippincott Raven, Philadelphia, 1997:31-94.

4. Chiappa KH. Principles of Evoked Potentials In Chiappa K H (ed). Evoked potentials in clinical medicine. Philadelphia: LippincottRaven,1997:1-30.

5. Goodin DS. Clinical utilility cognitive event related potentials. EEG Clin Neurophysiol 1990;76:2-5.

6. Goodin DS, Starr A, Chippendale P, Squires KC. Sequential changes in P3 component of the auditory evoked potential in confusional states and dementing illnesses. Neurology1983,33:1215-1218.

7. Sutton NS, Braren M, John ER. Evoked potentials correlates of stimulus uncertainty. Science 1965;150:1187-1188.

8. Brown WS, Marsh JT, LaRue A. Event related potentials in psychiatry: differenting depression and dementia in elderly. Bull. Los Angeles Neurol Soc 1982;47:91-107.

9. Goodin DS, Squires KC, Starr, A. Long latency event related components of the auditory evoked potential in dementia. Brain 1978;101:635-648.

10. Goodin DS, Squires KC, Henderson BH, Starr A. Aged related variations in evoked potentials to auditory stimuli in normal human subjects. EEG Clin Neurophysiol 1978;44:447-458.

11. Brown WS, Marsh JT, LaRue A. A Exponential electrophysiological aging: P300 latency. EEG Clin Neurophysiol 1983;55:277-295. 